\title{
Avaliação de macro e microminerais em frutas tropicais cultivadas no nordeste brasileiro
}

\author{
Evaluation of macro and micro-mineral content in tropical fruits cultivated in the northeast of Brazil
}

\author{
Maria Mozarina Beserra ALMEIDA ${ }^{1,2 *}$, Paulo Henrique Machado de SOUSA ${ }^{3}$, Maria Luciana FONSECA ${ }^{1}$, \\ Carlos Emanuel Carvalho MAGALHÃES ${ }^{4}$, Maria de Fátima Gomes LOPES ${ }^{1}$, Telma Leda Gomes de LEMOS$^{2}$
}

\begin{abstract}
Resumo
O Brasil tem grande importância no mercado de frutas; porém há escassez de dados acerca da composição das frutas tropicais brasileiras, principalmente daquelas produzidas no nordeste. No presente estudo, determinaram-se macro e microminerais de 11 frutas tropicais cultivadas no nordeste brasileiro: abacaxi, ata, graviola, jaca, mamão, mangaba, murici, sapoti, seriguela, tamarindo e umbu. As amostras foram desidratadas e mineralizadas em $\mathrm{HNO}_{3} / \mathrm{HClO}_{4}$ (3:1). Os minerais $\mathrm{Ca}, \mathrm{Mg}, \mathrm{Mn}, \mathrm{Zn}, \mathrm{Fe}, \mathrm{Co}$, Se e Ni foram analisados por espectrofotometria de absorção atômica, $\mathrm{Na}$ e $\mathrm{K}$ por fotometria de chama e $\mathrm{P}$ por método colorimétrico. Os resultados indicaram que o tamarindo pode ser considerado excelente fonte de $\mathrm{Mg}, \mathrm{Cu}$ e $\mathrm{K}$; além de boa fonte em $\mathrm{Ca}, \mathrm{P}, \mathrm{Fe}$ e Se. Dentre os minerais avaliados nas frutas estudadas, $\mathrm{K}$ apresentou o maior conteúdo, seguido pelo Ca e Mg. Ata, graviola, sapoti e murici são boas fontes de dois ou mais minerais. Correlações elevadas foram obtidas entre K e os minerais P, Co e Fe; e entre Co e Fe. Portanto, sugere-se o consumo dos frutos tropicais estudados, como auxiliares na reposição de nutrientes minerais.
\end{abstract}

Palavras-chave: frutas tropicais; macrominerais; microminerais; ingestão diária recomendada; correlação de Pearson.

\begin{abstract}
Brazil has great importance in the fruits market; however, data on the composition of Brazilian tropical fruits, mainly of those produced in the Northeast region, is scarce. In the present study, it was determined the macro- and micro-minerals of 11 tropical fruits cultivated in the Northeast of Brazil: pineapple, sweetsop, soursop, jackfruit, papaya, mangaba, murici, sapodilla, ciruela, tamarind, and umbu. The samples were dehydrated and mineralized in $\mathrm{HNO}_{3} / \mathrm{HClO}_{4}(3: 1)$ solution. The minerals $\mathrm{Ca}, \mathrm{Mg}, \mathrm{Mn}, \mathrm{Zn}, \mathrm{Fe}, \mathrm{Co}$, Se and Ni were analyzed by atomic absorption spectrophotometry; $\mathrm{Na}$ and $\mathrm{K}$ by flame photometry; and $\mathrm{P}$ by the colorimetric method. The results indicated that tamarind is a rich source of all minerals available, especially of $\mathrm{Mg}, \mathrm{Cu}$ and $\mathrm{K}$, in addition to being a good source of $\mathrm{Ca}, \mathrm{P}, \mathrm{Fe}$, and $\mathrm{Se}$. Among the minerals evaluated in this study, $\mathrm{K}$ presented the highest content, followed by $\mathrm{Ca}$ and $\mathrm{Mg}$. Sweetsop, soursop, sapodilla, and murici are good sources of two or more minerals. High correlations were obtained between $\mathrm{K}$ and the minerals $\mathrm{P}$, Co, and Fe and between Co and Fe. Therefore, the consumption of the studied tropical fruits is suggested as an assistant to the reposition of mineral nutrients.
\end{abstract}

Keywords: tropical fruits; macro-minerals; micro-minerals; dietary reference intakes; Pearson's correlation.

\section{Introdução}

Muitos problemas atuais de saúde estão relacionados aos padrões dietéticos assumidos pelos indivíduos. Segundo a Organização Mundial de Saúde (OMS), a má nutrição, que inclui não apenas a subnutrição mas também as deficiências específicas e o excesso de ingestão de alimentos, continua persistindo em todos os países. Dados da OMS indicam que enquanto 800 milhões de pessoas não chegam a cobrir suas necessidades básicas de energia e proteína, outro contingente de 600 milhões sofre com as consequências de uma alimentação inadequada. Portanto, a deficiência nutricional é uma das maiores preocupações para muitos países em desenvolvimento, necessitando atenção do ponto de vista da saúde pública. Estudos estabelecem que a ingestão regular de frutas e verduras diminui o risco de doenças cardiovasculares, câncer, diabetes, hipertensão (LETERME et al., 2006; RIMM et al., 2002).

Nos últimos tempos, deu-se uma maior intensificação nos estudos de micronutrientes, tanto em nações desenvolvidas como em desenvolvimento, por acreditar-se que muitos problemas de saúde estão relacionados, pelo menos em parte, à insuficiência de determinados micronutrientes. Estudos comprovam a riqueza nutricional em frutas e verduras de constituintes como: vitaminas A e C, minerais, fibras e vários fitoquímicos (SMOLIN; GROSVENOR, 2007; MILTON, 2003). A importância da inclusão de minerais na dieta têm sido amplamente discutida em textos sobre nutrição

Recebido para publicação em 28/11/2007

Aceito para publicação em 14/1/2008 (003042)

${ }^{1}$ Departamento de Química Analítica e Físico-Química, CP 12168, CEP 60356-000, Fortaleza - CE, Brasil, E-mail: mozaba@ufc.br

2 Curso de Pós-Graduação em Química Orgânica, Universidade Federal do Ceará - UFC, CP 12168, CEP 60356-000, Fortaleza-CE, Brasil, E-mail: tlemos@dqoi.ufc.br

${ }_{3}^{3}$ Departamento de Tecnologia de Alimentos, Universidade Federal do Ceará - UFC, Campus do Pici, Av. Mister Hull, 2977, CP 12168, CEP 60356-000, Fortaleza - CE, Brasil,

E-mail: phenriquemachado@gmail.com

${ }^{4}$ Centro de Ciências e Tecnologia, Universidade Estadual do Ceará - UFC, Campus do Itaperi, Av. Paranjana, 1700, CEP 60740-903, Fortaleza - CE, Brasil,

E-mail:cecmagal@yahoo.com.br

${ }^{*}$ A quem a correspondência deve ser enviada 
(GONCALVES; TEODORO; TAKASE, 2007; FRANCO, 1999; BERDANIER, 1998).

Baseado na produção de 32 milhões de toneladas de frutas em uma área plantada de dois milhões de hectares, o Brasil é considerado o terceiro maior produtor de frutas do mundo, sobrepondo-se a países como Estados Unidos, Itália e Espanha, ficando atrás apenas da China e Índia (MAIA; SOUSA; LIMA, 2007; KUSKOSKI et al., 2005). Além disso, observa-se que um grande número de frutas tropicais é comestível e que, paradoxalmente, poucas espécies estão disponíveis no mercado. Esse fato pode estar relacionado à baixa disponibilidade de algumas frutas, desconhecimento sobre os sistemas de produção e conservação, bem como aspectos relacionados à qualidade das frutas (LETERME et al., 2006). Estudos sobre a caracterização química das frutas tropicais são limitados, existindo ainda uma grande variedade dessas frutas com composição química desconhecida. Portanto, esse trabalho objetivou estudar os teores de macrominerais $(\mathrm{Na}, \mathrm{K}, \mathrm{Mg}, \mathrm{Ca}, \mathrm{P})$ e microminerais ( $\mathrm{Fe}, \mathrm{Mn}, \mathrm{Cu}, \mathrm{Zn}, \mathrm{Ni}$, Co e Se) em 11 espécies de frutas cultivadas no nordeste brasileiro.

\section{Material e métodos}

\subsection{Amostras}

As amostras das frutas foram coletadas nas proximidades da cidade de Fortaleza, Ceará, Brasil, de janeiro a março de 2006, autenticadas pelo Prof. Dr. Edson Paula Numes (Departamento de Biologia, Universidade Federal do Ceará) e suas exsicatas encontram-se depositadas no Herbário Prisco Bezerra da Universidade Federal do Ceará. O nome popular, o nome científico e a família de cada fruta estão apresentados na Tabela 1.

\subsection{Tratamento das amostras}

As amostras, depois de lavadas com água corrente e água destilada, foram secas à temperatura ambiente e submetidas a processo de extração das polpas, separando-as das cascas e sementes, à exceção de seriguela e umbu, nas quais a pele foi mantida. O material assim obtido para cada fruta (cerca de $2,5 \mathrm{~g}$ ) foi então dessecado $\left(105^{\circ} \mathrm{C}\right)$ e mineralizado em solução digestora composta por ácido nítrico e ácido perclórico na proporção

Tabela 1. Nomes populares, científicos e famílias das espécies de frutas tropicais estudadas.

\begin{tabular}{lll}
\hline Nome popular & \multicolumn{1}{c}{ Nome científico } & \multicolumn{1}{c}{ Família } \\
\hline Abacaxi & Ananas comosus (L.) Merr. & Bromeliaceae \\
Ata & Annona squamosa L. & Annonaceae \\
Graviola & Annona muricata $\mathrm{L}$. & Annonaceae \\
Jaca & Artocarpus integrifolia $\mathrm{L}$. & Moraceae \\
Mamão & Carica papaya L. & Caricaceae \\
Mangaba & Hancornia speciosa Gomes & Apocynaceae \\
Murici & Byrsonima crassifolia (L.) Kunth & Malpighiaceae \\
Sapoti & Manilkara zapota (L.) P. Royen & Sapotaceae \\
Serigüela & Spondias purpurea L. & Anacardiaceae \\
Tamarindo & Tamarindus indica L. & Caesalpiniaceae \\
Umbu & Spondias tuberosa Arruda Câmara & Anacardiaceae \\
\hline
\end{tabular}

$\mathrm{HNO}_{3}: \mathrm{HClO}_{4}(3: 1 \mathrm{v} / \mathrm{v})$, conforme descrito por Silva (1999). As misturas foram adicionadas a tubos digestores, onde permaneceram durante toda noite em temperatura ambiente. Em seguida, a temperatura foi aumentada gradativamente por 12 horas até $220^{\circ} \mathrm{C}$, utilizando bloco digestor (TECNAL modelo TE007D V), até redução de volume para aproximadamente $1,5 \mathrm{~mL}$. Os tubos foram esfriados à temperatura ambiente e a solução transparente resultante foi filtrada, diluída com água deionizada para $50 \mathrm{~mL}$ e usada para análise dos minerais.

\subsection{Determinações dos teores dos elementos minerais}

Os macronutrientes ( $\mathrm{Ca}$ e $\mathrm{Mg}$ ) e os micronutrientes ( $\mathrm{Mn}$, $\mathrm{Zn}, \mathrm{Fe}, \mathrm{Co}, \mathrm{Se}$ e Ni) foram determinados quantitativamente por espectrometria de absorção atômica. Para os minerais Mn, Zn, $\mathrm{Fe}, \mathrm{Ca}$ e Mg, foi utilizado um espectrômetro com chama, modelo ATOMICA-GBC 933 PLUS, calibrado em condições específicas de comprimento de onda, fenda e mistura dos gases para cada elemento. Na quantificação de $\mathrm{Co}$, Se e Ni utilizou-se um espectrômetro VARIAN, Modelo SpectrAA 220, com sistema de correção de fundo por efeito Zeeman longitudinal, equipado com forno de grafite como atomizador e lâmpada de catodo oco como fonte de radiação, seguindo o programa de aquecimento do tubo recomendado pelo fabricante. Para a construção das curvas de calibração, foram utilizadas ampolas de padrões para absorção atômica Merck, devidamente diluídas com água deionizada. Os macronutrientes $\mathrm{Na}$ e $\mathrm{K}$ foram analisados por fotometria de chama (ANALYSER, 910M), enquanto o P foi determinado por espectrofotometria de absorção molecular (Método Azul de Molibdênio), utilizando um espectrofotômetro UV-visível, MICRONAL, modelo B582. Todas as análises foram realizadas em triplicata. As condições operacionais das análises são sumarizadas na Tabela 2.

\subsection{Análises estatísticas}

As relações entre os conteúdos dos minerais foram averiguadas através do teste de correlação de Pearson, usando o programa estatístico SAEG (2007), versão 9.1.

Tabela 2. Condições operacionais utilizadas para a determinação de minerais em frutos tropicais do nordeste brasileiro.

\begin{tabular}{ccccc}
\hline Elemento & Técnica & $\lambda_{\text {nm }}$ & Faixa linear & Referência \\
\hline $\mathrm{Na}$ & FC & 589,0 & - & AOAC (1980) \\
$\mathrm{K}$ & $\mathrm{FC}$ & 766,5 & - & AOAC (1980) \\
$\mathrm{Ca}$ & EAAC & 422,7 & $1,0-5,0 \mathrm{mg} \cdot \mathrm{L}^{-1}$ & Welz $(1999)$ \\
$\mathrm{Mg}$ & EAAC & 285,2 & $0,1-0,5 \mathrm{mg} \cdot \mathrm{L}^{-1}$ & Welz (1999) \\
$\mathrm{P}$ & EAM & 660,0 & $0,03-0,4 \mathrm{mg} \cdot \mathrm{L}^{-1}$ & Silva (1999) \\
$\mathrm{Fe}$ & EAAC & 248,3 & $1,0-5,0 \mathrm{mg} \cdot \mathrm{L}^{-1}$ & Christian (2003) \\
$\mathrm{Mn}$ & EAAC & 279,5 & $1,0-5,0 \mathrm{mg} \cdot \mathrm{L}^{-1}$ & Welz (1999) \\
$\mathrm{Cu}$ & EAAC & 324,7 & $0,03-10 \mathrm{mg} . \mathrm{L}^{-1}$ & Welz (1999) \\
$\mathrm{Zn}$ & EAAC & 213,9 & $0,1-0,4 \mathrm{mg} \cdot \mathrm{L}^{-1}$ & Welz (1999) \\
$\mathrm{Ni}$ & EAAFG & 232,5 & $0-33 \mu \mathrm{g} \cdot \mathrm{L}^{-1}$ & Welz (1999) \\
$\mathrm{Co}$ & EAAFG & 242,5 & $0-35 \mu \mathrm{g} \cdot \mathrm{L}^{-1}$ & Welz (1999) \\
$\mathrm{Se}$ & EAAFG & 196,2 & $0-33 \mu \mathrm{g} . \mathrm{L}^{-1}$ & Welz (1999)
\end{tabular}

EAAC: Espectrofotometria de absorção atômica com chama; EAAFG: espectrofotometria de absorção atômica com forno de grafite; EAM: espectrofotometria de absorção molecular; e FC: fotometria de chama. 


\section{Resultados e discussão}

Na Tabela 3 são mostrados os teores de macrominerais de frutos tropicais do nordeste brasileiro, bem como a comparação destes valores com dados da literatura. Na Tabela 4 novamente é feita essa comparação, considerando-se agora os resultados encontrados para os sete microminerais investigados. Os conteúdos de $\mathrm{K}$ foram os mais elevados, variando de 143,67 a 790,11 mg.100 g $\mathrm{g}^{-1}$ de porção comestível, seguidos de Ca (8,52-127,66 mg.100 g $\left.\mathrm{g}^{-1}\right)$ e $\mathrm{Mg}\left(3,21-70,27 \mathrm{mg} .100 \mathrm{~g}^{-1}\right)$, enquanto que teores de Se foram os mais baixos, oscilando de 0,11 a $2,36 \mu \mathrm{g} \cdot 100 \mathrm{~g}^{-1}$. Com os resultados obtidos, foi possível calcular as contribuições percentuais dos minerais nas amostras das frutas analisadas em relação à Ingestão Diária Recomendada (IDR) para um adulto (BRASIL, 2005), conforme apresentado na Tabela 5. Para Na e K foram usados os valores de Recommended Dietary Allowance (RDA) de 500 e 2000 mg para $\mathrm{Na}$ e K, respectivamente (BERDANIER, 1998). As frutas foram classificadas de acordo com a definição da FDA (Food and Drug
Administration) em "excelentes" ou "boas" fontes de nutrientes quando uma porção ingerida da fruta pode suprir pelo menos 20 e 10-20\% da IDR, respectivamente (MILLER-IHLI, 1996).

De um modo geral, as frutas investigadas mostraram baixas concentrações de $\mathrm{Na}$ e a maioria delas também apresentou baixos teores de $\mathrm{Ca}$, destacando-se apenas as amostras de sapoti e tamarindo por apresentaram teores de Ca suficientes para suprir 10\% da IDR em $100 \mathrm{~g}$ de porção comestível. Os teores de $\mathrm{Na}$ apresentaram, em média, a metade dos valores daqueles encontrados na tabela norte-americana da United States Departament of Agriculture (USDA, 2007), e em geral, um terço daqueles obtidos para abacaxi, ata, graviola e mamão cultivadas na Colômbia (LETERME et al., 2006). Em tabela reportada por Franco (1999) as concentrações de Na são ainda mais elevadas. Por exemplo, para o caso do mamão, essa tabela apresenta valor de $\mathrm{Na}$ dezesseis vezes maior que o encontrado em nosso estudo. As concentrações de Ca foram compatíveis com o estudo de frutas da Colômbia e da USDA, excetuando-se

Tabela 3. Teores de macrominerais em frutas tropicais do nordeste brasileiro e dados da literatura.

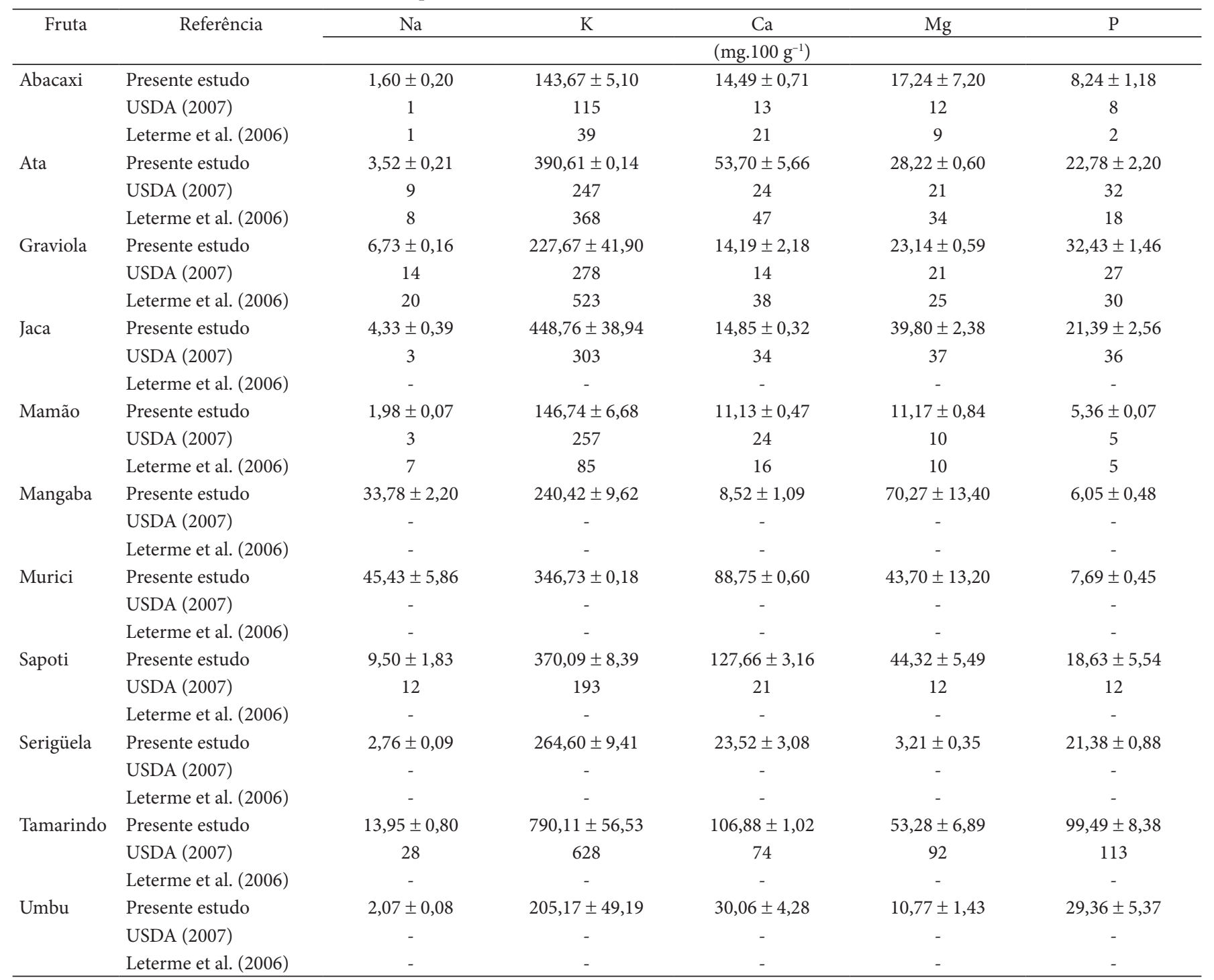


Tabela 4. Teores de microminerais em frutas tropicais do nordeste brasileiro e dados da literatura.

\begin{tabular}{|c|c|c|c|c|c|c|c|c|}
\hline \multirow[t]{2}{*}{ Fruta } & \multirow[t]{2}{*}{ Referência } & $\mathrm{Fe}$ & $\mathrm{Mn}$ & $\mathrm{Cu}$ & $\mathrm{Zn}$ & $\mathrm{Se}$ & Co & $\mathrm{Ni}$ \\
\hline & & \multicolumn{4}{|c|}{$\left(\mathrm{mg} .100 \mathrm{~g}^{-1}\right)$} & \multicolumn{3}{|c|}{$\left(\mu \mathrm{g} .100 \mathrm{~g}^{-1}\right)$} \\
\hline \multirow[t]{3}{*}{ Abacaxi } & Presente estudo & $0,69 \pm 0,07$ & $1,35 \pm 0,29$ & $0,14 \pm 0,07$ & $0,17 \pm 0,03$ & $0,81 \pm 0,10$ & $9,64 \pm 1,44$ & $44,62 \pm 0,56$ \\
\hline & USDA (2007) & 0,28 & 1,177 & 0,099 & 0,10 & 0,1 & - & - \\
\hline & Leterme et al. (2006) & 0,32 & 0,26 & 0,01 & 0,09 & $\mathrm{Nd}$ & nd & 110 \\
\hline \multirow[t]{2}{*}{ Ata } & Presente estudo & $0,75 \pm 0,17$ & $0,16 \pm 0,00$ & $0,22 \pm 0,03$ & $0,12 \pm 0,03$ & $0,26 \pm 0,11$ & $20,76 \pm 1,71$ & $19,42 \pm 0,72$ \\
\hline & USDA (2007) & 0,60 & - & 0,086 & 0,10 & 0,6 & - & - \\
\hline \multirow{2}{*}{ Graviola } & USDA (2007) & 0,60 & - & 0,086 & 0,10 & 0,6 & - & - \\
\hline & Leterme et al. (2006) & 0,38 & 0,07 & 0,10 & 0,11 & $\mathrm{Nd}$ & nd & 30 \\
\hline \multirow[t]{3}{*}{ Jaca } & Presente estudo & $0,79 \pm 0,04$ & $0,03 \pm 0,01$ & $0,23 \pm 0,02$ & $0,27 \pm 0,03$ & $0,36 \pm 0,01$ & $22,91 \pm 2,07$ & $32,77 \pm 2,69$ \\
\hline & USDA (2007) & 0,60 & 0,197 & 0,187 & 0,42 & 0,6 & - & - \\
\hline & Leterme et al. (2006) & - & - & - & - & - & - & - \\
\hline \multirow[t]{3}{*}{ Mangaba } & Presente estudo & $0,95 \pm 0,07$ & $\mathrm{Nd}$ & $0,08 \pm 0,04$ & $0,12 \pm 0,01$ & $0,80 \pm 0,04$ & $20,93 \pm 1,75$ & $13,66 \pm 0,54$ \\
\hline & USDA (2007) & - & - & - & - & - & - & - \\
\hline & Leterme et al. (2006) & - & - & - & - & - & - & - \\
\hline \multirow[t]{3}{*}{ Murici } & Presente estudo & $0,71 \pm 0,00$ & $0,08 \pm 0,01$ & $0,09 \pm 0,01$ & $0,09 \pm 0,06$ & $2,36 \pm 0,24$ & $27,24 \pm 1,26$ & $26,41 \pm 4,25$ \\
\hline & USDA (2007) & - & - & - & - & - & - & - \\
\hline & Leterme et al. (2006) & - & - & - & - & - & - & - \\
\hline \multirow[t]{3}{*}{ Sapoti } & Presente estudo & $0,83 \pm 0,06$ & $\mathrm{Nd}$ & $0,06 \pm 0,01$ & $0,09 \pm 0,07$ & $0,45 \pm 0,03$ & $30,65 \pm 1,94$ & $30,96 \pm 1,09$ \\
\hline & USDA (2007) & 0,80 & - & 0,086 & 0,10 & 0,6 & - & - \\
\hline & Leterme et al. (2006) & - & - & - & - & - & - & - \\
\hline \multirow[t]{2}{*}{ Serigüela } & Presente estudo & $0,50 \pm 0,01$ & $\mathrm{Nd}$ & $0,12 \pm 0,04$ & $0,31 \pm 0,07$ & $0,49 \pm 0,03$ & $13,11 \pm 0,75$ & $29,36 \pm 1,18$ \\
\hline & USDA (2007) & - & - & - & - & - & - & - \\
\hline
\end{tabular}

nd - não determinado.

Tabela 5. Valores de ingestão diária recomendada (IDR) de minerais (mg/dia ou $\mu \mathrm{g} / \mathrm{dia}$ ) para adultos e contribuição mineral para IDR (\%) em relação a $100 \mathrm{~g}$ de frutas tropicais do nordeste brasileiro.

\begin{tabular}{|c|c|c|c|c|c|c|c|c|c|c|c|c|}
\hline \multirow[t]{2}{*}{ Minerais } & \multirow[t]{2}{*}{ IDR } & \multicolumn{11}{|c|}{ Contribuição de minerais para IDR (\%) em relação a uma porção de $100 \mathrm{~g}$ de fruta } \\
\hline & & Abacaxi & Ata & Graviola & Jaca & Mamão & Mangaba & Murici & Sapoti & Serigüela & Tamarindo & Umbu \\
\hline $\mathrm{Ca}$ & $1000 \mathrm{mg}$ & 1,45 & 5,37 & 1,42 & 1,49 & 1,11 & 0,85 & 8,88 & 12,77 & 2,35 & 10,69 & 3,01 \\
\hline $\mathrm{Mg}$ & $260 \mathrm{mg}$ & 6,63 & 10,85 & 8,9 & 15,31 & 4,3 & 27,03 & 16,81 & 17,05 & 1,23 & 20,49 & 4,14 \\
\hline $\mathrm{P}$ & $700 \mathrm{mg}$ & 1,18 & 3,25 & 4,63 & 3,06 & 0,77 & 0,86 & 1,10 & 2,66 & 3,05 & 14,21 & 4,19 \\
\hline $\mathrm{Fe}$ & $14 \mathrm{mg}$ & 4,93 & 5,36 & 5,93 & 5,64 & 1,79 & 6,79 & 5,07 & 5,93 & 3,57 & 12,07 & 2,93 \\
\hline $\mathrm{Mn}$ & $2,3 \mathrm{mg}$ & 58,7 & 6,96 & 3,04 & 1,3 & - & - & 3,48 & - & - & 2,61 & - \\
\hline $\mathrm{Zn}$ & $7 \mathrm{mg}$ & 2,43 & 1,71 & 6,57 & 3,86 & 0,43 & 1,71 & 1,29 & 1,29 & 4,43 & 1,29 & 2,00 \\
\hline $\mathrm{Cu}$ & $900 \mu \mathrm{g}$ & 15,56 & 24,44 & 16,67 & 25,56 & 2,78 & 8,89 & 10,00 & 6,67 & 13,13 & 32,22 & 7,78 \\
\hline $\mathrm{Se}$ & $34 \mu \mathrm{g}$ & 2,38 & 0,76 & 2,32 & 1,06 & 1,15 & 2,35 & 6,94 & 1,32 & 1,44 & 9,21 & 0,32 \\
\hline
\end{tabular}

o sapoti que apresentou conteúdo cerca de seis vezes maior que o da USDA. Em relação ao teor de $\mathrm{Mg}$, mangaba e tamarindo podem ser consideradas excelentes fontes (mais do que $20 \%$ da IDR), enquanto sapoti, murici, jaca e ata, são boas fontes desse mineral por contribuírem com mais que $10 \%$ da IDR. Quanto ao teor de P, somente o tamarindo contribuiu com mais de $10 \%$ da IDR. Altos teores de $\mathrm{K}$ foram obtidos para a maioria das frutas, destacando-se tamarindo e jaca. Os resultados dos teores de $\mathrm{Mg}$, 
P e K são compatíveis com os encontrados na USDA (2007) e com as frutas estudadas por Leterme et al. (2006). Entretanto, o conteúdo de K para abacaxi e tamarindo é aproximadamente três vezes maior que aqueles reportados por Franco (1999).

Analisando os resultados para os micronutrientes, observou-se que as frutas que apresentaram os maiores e menores conteúdos de Fe são as mesmas encontradas no estudo da USDA, ou seja, tamarindo e mamão, respectivamente. Constatou-se ainda que a concentração de Fe presente no tamarindo supre $12 \%$ da IDR, sendo, portanto, essa uma boa fonte de Fe, e que em outras 7 frutas (mangaba, abacaxi, ata, graviola, jaca, murici e sapoti) foram encontrados teores mais baixos desse mineral. De modo geral, as frutas apresentaram baixos conteúdos de $\mathrm{Mn}, \mathrm{Zn}$ e Se, excetuando-se abacaxi, que demonstrou ser uma rica fonte de Mn (58,7\% da IDR), e tamarindo, que pode suprir quase $10 \%$ da IDR para o Se. A maioria das frutas investigadas indicou ser considerável fonte de $\mathrm{Cu}$, destacando-se tamarindo, jaca e ata como excelentes fontes desse mineral (24,4-32,2\% da IDR), e graviola, abacaxi, seriguela e murici como boas fontes. Observando-se a composição mineral do abacaxi nesse estudo, verificou-se que os teores de $\mathrm{Mn}$ e $\mathrm{Cu}$ foram pelo menos o dobro daqueles registrados para a mesma fruta cultivada nos Estados Unidos (MILLER-IHLI, 1996) ou na Colômbia (LETERME et al., 2006). No caso da mangaba, foi encontrado apenas um registro do teor de minerais em frutos do Estado do Mato Grosso do Sul, relatada por Hiane et al. (1992). Segundo esses pesquisadores, a mangaba apresentou mais altas concentrações de $\mathrm{P}$ e Zn, e conteúdos de $\mathrm{Mg}$ e Fe cerca de um terço daqueles encontrados no presente estudo. Dentre as amostras pesquisadas, tamarindo e abacaxi apresentaram-se como as maiores fontes de $\mathrm{Ni}$, enquanto que as maiores concentrações de Co foram encontradas no tamarindo, sapoti e murici.

Sabe-se que a composição mineral em frutas pode ser influenciada por vários fatores, como condições climáticas (luz, temperatura, umidade), composição química do solo, diferenças genéticas e práticas agrícolas (OLIVARES et al., 2004; HARDISSON et al., 2001; SANCHEZ-CASTILLO et al.,1998). Esses fatores podem ter contribuído para as diferenças encontradas entre o presente estudo e as referências consultadas.

Os coeficientes de correlação de Pearson entre as concentrações dos minerais e seus níveis de significância são apresentados na Tabela 6. Valores elevados de correlações foram observados $(\mathrm{p}<0,001)$ entre o $\mathrm{K}$ e os minerais $\mathrm{P}$, Co e $\mathrm{Fe}$; assim como entre Co e Fe. Correlações menores $(\mathrm{p}<0,01)$ também foram observadas, por exemplo, $(\mathrm{p}<0,01)$, entre os elementos $\mathrm{Na}$ e $\mathrm{Mg}(\mathrm{r}=0,69), \mathrm{K}$ e $\mathrm{Ni}(\mathrm{r}=0,77)$, Ca e Co $(0,76)$, entre o $\mathrm{P}$ e Co, Fe e Ni, e entre ferro e Ni e Se. Verificou-se que o Se apresentou correlação significativa com quase todos os elementos testados, com exceção do $\mathrm{Mg}$, Cu e Zn. Por outro lado, o Mn e o Zn não apresentaram correlação significativa ( $\mathrm{p}>0,05)$ com nenhum dos elementos testados, enquanto o $\mathrm{Na}$ apresentou-se quase independente, apresentando correlação significativa somente com o $\mathrm{Mg}$ e Se, fato também observado por Leterme et al. (2006), em um estudo com conteúdo mineral em frutas tropicais, em que não verificou correlação entre o sódio e outros 10 minerais testados $(\mathrm{Ca}, \mathrm{P}, \mathrm{K} . \mathrm{Mg}, \mathrm{Cl}, \mathrm{S}, \mathrm{Mn}$, $\mathrm{Zn}, \mathrm{Fe}$ e $\mathrm{Cu}$ ). Entretanto, raras são as informações disponíveis na literatura sobre essas correlações em frutas.

\section{Conclusões}

Dentre os 12 minerais ( $\mathrm{Na}, \mathrm{Ni}, \mathrm{Co}, \mathrm{Se}, \mathrm{Ca}, \mathrm{Mg}, \mathrm{Fe}, \mathrm{P}, \mathrm{Cu}$, $\mathrm{Zn}, \mathrm{K}, \mathrm{Mn}$ ) estudados nas 11 espécies de frutas tropicais, o K é o mineral mais abundante $(143,67$ a 790,11 mg.100 g-1), seguido por $\mathrm{Ca}\left(8,52\right.$ a $\left.127,66 \mathrm{mg} .100 \mathrm{~g}^{-1}\right)$ e $\mathrm{Mg}(3,21$ a $\left.70,27 \mathrm{mg} .100 \mathrm{~g}^{-1}\right)$.

Considerando-se o consumo diário recomendado para os minerais estudados, ata, graviola, sapoti e murici podem ser classificados como boas fontes de pelo menos dois deles, dentre $\mathrm{K}, \mathrm{Mg}, \mathrm{Cu}$, Ca, por prover de 10 a $19 \%$ da IDR. Mangaba e abacaxi são, respectivamente, excelentes fontes de $\mathrm{Mg}$ (70,27 mg.100 g $\mathrm{g}^{-1}$ ) e Mn (1,35 mg.100 g-1), enquanto ata e jaca destacam-se por seus elevados teores de $\mathrm{Cu}(0,22 \mathrm{e}$ $0,23 \mathrm{mg} .100 \mathrm{~g}^{-1}$, respectivamente), satisfazendo, todas elas, mais do que $20 \%$ de IDR. Conteúdos encontrados de Na e Zn foram

Tabela 6. Correlação linear (r) entre concentrações de elementos nas frutas tropicais do nordeste brasileiro e nível de significância de correlação de acordo com análise de Pearson.

\begin{tabular}{|c|c|c|c|c|c|c|c|c|c|c|c|}
\hline & $\mathrm{Na}$ & $\mathrm{K}$ & $\mathrm{Ca}$ & $\mathrm{Mg}$ & $\mathrm{P}$ & Co & $\mathrm{Fe}$ & $\mathrm{Ni}$ & $\mathrm{Cu}$ & $\mathrm{Mn}$ & $\mathrm{Zn}$ \\
\hline K & $0,15^{\mathrm{NS}}$ & - & - & - & - & - & - & - & - & - & - \\
\hline $\mathrm{Ca}$ & $0,30^{\mathrm{NS}}$ & $0,64^{\star}$ & - & - & - & - & - & - & - & - & - \\
\hline $\mathrm{Mg}$ & $0,69^{* *}$ & $0,52^{\mathrm{NS}}$ & $0,40^{\mathrm{NS}}$ & - & - & - & - & - & - & - & - \\
\hline $\mathrm{P}$ & $-0,12^{\mathrm{NS}}$ & $0,83^{* * *}$ & $0,44^{\mathrm{NS}}$ & $0,21^{\mathrm{NS}}$ & - & - & - & - & - & - & - \\
\hline Co & $0,36^{\mathrm{NS}}$ & $0,94^{* * *}$ & $0,76^{* *}$ & $0,68^{*}$ & $0,76^{* *}$ & - & - & - & - & - & - \\
\hline $\mathrm{Fe}$ & $0,28^{\mathrm{NS}}$ & $0,85^{* * *}$ & $0,51^{\mathrm{NS}}$ & $0,70^{* *}$ & $0,79^{* *}$ & $0,91^{* * *}$ & - & - & - & - & - \\
\hline $\mathrm{Ni}$ & $-0,01^{\mathrm{NS}}$ & $0,77^{\star *}$ & $0,50^{\mathrm{NS}}$ & $0,29^{\mathrm{NS}}$ & $0,76^{\star *}$ & $0,76^{* *}$ & $0,80^{* *}$ & - & - & - & - \\
\hline $\mathrm{Cu}$ & $-0,16^{\mathrm{NS}}$ & $0,77^{\star *}$ & $0,16^{\mathrm{NS}}$ & $0,24^{\mathrm{NS}}$ & $0,70^{* *}$ & $0,59^{*}$ & $0,71^{\star *}$ & $0,71^{* *}$ & - & - & - \\
\hline $\mathrm{Mn}$ & $-0,25^{\mathrm{NS}}$ & $-0,43^{\mathrm{NS}}$ & $-0,34^{\mathrm{NS}}$ & $-0,37^{\mathrm{NS}}$ & $-0,34^{\mathrm{NS}}$ & $-0,37^{\mathrm{NS}}$ & $-0,15^{\mathrm{NS}}$ & $0,23^{\mathrm{NS}}$ & $-0,13^{\mathrm{NS}}$ & - & - \\
\hline $\mathrm{Zn}$ & $-0,28^{\mathrm{NS}}$ & $-0,16^{\mathrm{NS}}$ & $-0,43^{\mathrm{NS}}$ & $-0,29^{\mathrm{NS}}$ & $0,03^{\mathrm{NS}}$ & $-0,23^{\mathrm{NS}}$ & $-0,03^{\mathrm{NS}}$ & $-0,05^{\mathrm{NS}}$ & $0,24^{\mathrm{NS}}$ & $-0,09^{\mathrm{NS}}$ & - \\
\hline $\mathrm{Se}$ & $0,57^{\star}$ & $0,68^{*}$ & $0,54^{*}$ & $0,48^{\mathrm{NS}}$ & $0,62^{\star}$ & $0,78^{* *}$ & $0,73^{* *}$ & $0,71^{\star *}$ & $0,41^{\mathrm{NS}}$ & $-0,11^{\mathrm{NS}}$ & $-0,22^{\mathrm{NS}}$ \\
\hline
\end{tabular}

NS = não significativo; ${ }^{\star}$ significativo ao nível de $5 \%$ de probabilidade $(\mathrm{p}<0,05)$; ${ }^{*}$ significativo ao nível de $1 \%$ de probabilidade $(\mathrm{p}<0,01)$; e ${ }^{\star * *}$ significativo ao nível de $0,1 \%$ de probabilidade $(\mathrm{p}<0,001)$. 
menores que $10 \%$ da IDR, indicando não serem as amostras analisadas boas fontes destes elementos.

Portanto, as frutas estudadas podem ser consideradas fontes valiosas de muitos minerais, destacando-se o tamarindo, por ser o mais rico em praticamente todos os nutrientes analisados, sendo excelente fonte de $\mathrm{Mg}, \mathrm{Cu}, \mathrm{K}$ (20 a 39\% da IDR), além de ser boa fonte de Ca, P, Fe, Se ( 9 a 14\% da IDR). Assim, sugere-se a incorporação das frutas investigadas mais frequentemente à dieta da população nordestina.

Verificou-se correlação altamente significativa entre alguns minerais estudados: entre o $\mathrm{K}$ e os minerais $\mathrm{P}$, Co e Fe; assim como entre Co e Fe. Constatou-se, ainda, que o Se apresentou correlação significativa com quase todos os elementos testados; enquanto $\mathrm{Mn}$ e $\mathrm{Zn}$ não apresentaram correlação significativa com nenhum dos elementos testados.

\section{Agradecimentos}

Os autores agradecem aos órgãos de fomento CNPq, CAPES e FUNCAP pelo apoio financeiro recebido.

\section{Referências bibliográficas}

Association of Official Analytical Chemistry - AOAC. Official methods of analysis. 13 ed. Washington, D.C., 1980.

Berdanier, C. D.; FAIlla, M. L. Advanced Nutrition Micronutrients. Boca Raton, Flórida: CRC Press LLC, 1998. p. 236.

BRASIL. Agência Nacional de Vigilância Sanitária - ANVISA. Ministério da Saúde. RDC no 269, de 22 de setembro de 2005. Aprova o regulamento técnico sobre a Ingestão Diária Recomendada (IDR) de proteína, vitaminas e minerais. Diário Oficial da União, Poder Executivo, Brasília, DF, 23 de setembro de 2005.

CHRISTIAN, G. D. Analytical Chemistry. 6 ed. New York: John Wiley \& Sons, 2003. 848 p.

FRANCO, G. Tabela de composição química dos alimentos. 9 ed. Rio de Janeiro: Ed. Livraria Atheneu, 1999. p. 307.

GONÇALVES, E. C. B. A.; TEODORO, A. J.; TAKASE I. Teores de cobre em extratos de carne in natura e processada. Ciência e Tecnologia de Alimentos, v. 27, n. 2, p. 298-302, 2007.
HARDISSON, A. et al. Mineral composition of the banana (Musa acuminata) from the island of Tenerife. Food Chemistry, v. 73, n. 2, p. 153-161, 2001.

HIANE, P. A. et al. Teores de minerais de alguns frutos do Estado de Mato Grosso do Sul. Boletim do Centro de Pesquisa e Processamento de Alimentos, v. 10, n. 2, p. 208-214, 1992.

KUSKOSKI, E. M. et al. Aplicación de diversos métodos químicos para determinar actividad antioxidante en pulpa de frutos. Ciência e Tecnologia de Alimentos, v. 25, n. 4, p. 726-732, 2005.

LETERME, P. et al. Mineral content of tropical fruits and unconventional foods of the Andes and the rain forest of Colombia. Food Chemistry, v. 95, n. 4, p. 644-652, 2006.

MAIA, G. A.; SOUSA, P. H. M.; LIMA, A. S. Processamento de sucos de frutas tropicais. 1 ed. Fortaleza: Edições UFC, 2007. 320 p.

MILLER-IHLI, N. J. Atomic Absorption and Atomic Emission Spectrometry for the determination of the trace element content of selected fruits consumed in the United States. Journal of Food Composition and Analysis, v. 9, n. 4, p. 301-311, 1996.

MILTON, K. Micronutrient intakes of wild primates: are humans different? Comparative biochemistry and Physiology, v. 136A, n. 1, p. 47-59, 2003.

National Nutrient Database for Standard - USDA. Release 20 (2007). Disponível em: <http://www.nal.usda.gov/fnic/foodcomp/cgi-bin/ list_nut_edit.pl $>$. Acesso em: 08 novembro 2007.

OLIVARES, M. et al. Iron, Zinc, and Copper: Contents in Common Chilean Foods and Daily Intakes in Santiago, Chile. Nutrition, v. 20, n. 2, p. 205-212, 2004.

RIMM, E.B. Fruit and vegetables - building a solid foundation. The American Journal of Clinical Nutrition, v. 76, n. 1, p. 1-2, 2002.

SAEG. Sistema para Análises Estatísticas. Versão 9.1. Viçosa: Fundação Arthur Bernardes, 2007.

SANCHEZ-CASTILLO, C. P. et al. The Mineral Content of Mexican Fruits and Vegetables. Journal of Food Composition and Analysis, v. 11, n. 4 , p. $340-356,1998$.

SILVA, F. C. Manual de análises químicas de solos, plantas e fertilizantes. Brasília: Embrapa Comunicação para Transferência de Tecnologia/ Embrapa Solos/ Embrapa Informática para Agricultura, 1999. $370 \mathrm{p}$.

SMOLIN, L. A.; GROSVENOR, M. B. Nutrition: science and applications with bloklet package. 1 ed. Orlando: John Wiley \& Sons Inc, 2007. 864 p.

WELZ, B. Atomic absorption spectrometry. 3 ed. Deerfield: VCH, 1999. 965p. 